\title{
Rosiglitazone enhances glucose uptake in glomerular podocytes using the glucose transporter GLUT1
}

\author{
R. Lennon • G. I. Welsh • A. Singh • S. C. Satchell • \\ R. J. Coward • J. M. Tavaré • P. W. Mathieson • \\ M. A. Saleem
}

Received: 17 April 2009/Accepted: 14 May 2009 / Published online: 17 June 2009

(C) Springer-Verlag 2009

\begin{abstract} however, the mechanisms underlying this effect are PPAR $\gamma$ agonist rosiglitazone on human podocytes. 3T3-L1 adipocytes, nephrin-deficient podocytes, human the plasma membrane expression of GLUT1 was determined with bis-mannose photolabelling. translocation of GLUT1 to the plasma membrane. This

R. Lennon • G. I. Welsh • A. Singh · S. C. Satchell •

R. J. Coward · P. W. Mathieson · M. A. Saleem $(\bowtie)$

Academic and Children's Renal Unit,

Paul O'Gorman Lifeline Centre, University of Bristol,

Southmead Hospital,

Bristol BS10 5NB, UK

e-mail: M.Saleem@bristol.ac.uk

J. M. Tavaré

Department of Biochemistry, University of Bristol,

Bristol, UK

Aims/hypothesis Peroxisome proliferator-activated receptor (PPAR) $\gamma$ agonists are used increasingly in the treatment of type 2 diabetes. In the context of renal disease, PPAR $\gamma$ agonists reduce microalbuminuria in diabetic nephropathy; unknown. Glomerular podocytes are newly characterised insulin-sensitive cells and there is good evidence that they are targeted in diabetic nephropathy. In this study we investigated the functional and molecular effects of the

Methods Conditionally immortalised human podocytes were cultured with rosiglitazone and functional effects were measured with glucose-uptake assays. The effect of rosiglitazone on glucose uptake was also measured in glomerular endothelial cells, proximal tubular cells and podocytes treated with the NEFA palmitate. The role of the glucose transporter GLUT1 was investigated with immunofluorescence and small interfering RNA knockdown and

Results Rosiglitazone significantly increased glucose uptake in wild-type podocytes and this was associated with effect was blocked with GLUT1 small interfering RNA. Nephrin-deficient podocytes, glomerular endothelial cells and proximal tubular cells did not increase glucose uptake in response to either insulin or rosiglitazone. Furthermore, rosiglitazone significantly increased basal and insulinstimulated glucose uptake when podocytes were treated with the NEFA palmitate.

Conclusions/interpretation In conclusion, rosiglitazone has a direct and protective effect on glucose uptake in wild-type human podocytes. This represents a novel mechanism by which PPAR $\gamma$ agonists may improve podocyte function in diabetic nephropathy.

Keywords Diabetic nephropathy - Glucose transport . Insulin resistance $\cdot$ Microalbuminuria $\cdot$ Nephrin Podocyte . $\operatorname{PPAR} \gamma$ agonist $\cdot$ Rosiglitazone
Abbreviations
PAN Puromycin aminonucleoside
PPAR Peroxisome proliferator-activated receptor
siRNA Small interfering RNA

\section{Introduction}

The peroxisome proliferator-activated receptors (PPARs) are a family of nuclear receptors which were first described in 1990 [1]. There are three subtypes $(\alpha, \beta / \delta$ and $\gamma)$ with diverse roles in cellular metabolism [2] and equally diverse associations with disease states [3]. PPAR agonists are used therapeutically and the thiazolidinedione group of PPAR $\gamma$ agonists have made an important contribution to the treatment of type 2 diabetes over the past 10 years [4, 5]. A recent international clinical trial demonstrated that 
rosiglitazone in combination with lifestyle changes significantly reduced the risk of developing type 2 diabetes in susceptible individuals [6].

In the context of renal disease, PPAR $\gamma$ agonists have been shown to reduce microalbuminuria in patients with diabetic nephropathy [7-10], although the mechanisms for this are not fully understood. Regulation of renal filtration occurs at the level of the glomerular filtration barrier, which is comprised of endothelial cells, podocytes and an intervening glomerular basement membrane. In diabetic nephropathy there is disruption to this barrier with the consequent loss of protein into the urine. An early marker of this state is microalbuminuria. There is now good evidence that glomerular podocytes are directly targeted in diabetic nephropathy with loss of podocytes into the urine [11] and reduced production of the key podocyte protein nephrin [12]. These phenomena may be mechanisms that lead to disruption of the glomerular filtration barrier and to disease progression in diabetic nephropathy.

The clinical observations that PPAR $\gamma$ agonists reduce microalbuminuria in diabetic nephropathy indicate that these agents have direct effects at the level of the glomerular filtration barrier. Indeed, there is evidence of direct effects of these agents on podocytes. It has been shown that nephrin is upregulated in podocytes in response to PPAR $\gamma$ agonists [13] and that this effect occurs via direct action of the peroxisome proliferator response elements on the nephrin promoter. Furthermore, PPAR $\gamma$ agonists reduce podocyte apoptosis [14] and have been shown to be protective against the progression to glomerulosclerosis $[14,15]$ that is a feature of advanced diabetic nephropathy. More recently, the PPAR $\gamma$ agonist rosiglitazone was shown to prevent diabetic nephropathy in a mouse model of diabetes [16] and this was associated with a reduction in markers of oxidative injury. Together these findings indicate that PPAR $\gamma$ agonists have diverse effects on podocyte function; additional investigation is required to further define the pathways involved.

We have recently reported that human glomerular podocytes are insulin-sensitive cells and are able to rapidly transport glucose using the glucose transporters GLUT4 and GLUT1 [17]. In addition, we have shown that this effect depends on the production of nephrin, which is required for GLUT1 translocation, in podocytes, [18]. These observations demonstrate that insulin sensitivity in podocytes is uniquely controlled at a molecular level. We hypothesise that, in insulin-resistant states such as type 2 diabetes, the insulin sensitivity of podocytes is disrupted leading to adverse effects on podocyte function that predispose to nephropathy.

In this study we investigated the effect of rosiglitazone on podocyte glucose uptake and insulin sensitivity. We investigated the roles of the glucose transporter GLUT1 and the key podocyte protein nephrin. To relate these studies to a disease state, we used the NEFA palmitate to induce insulin resistance in podocytes.

\section{Methods}

Materials Cell culture plastics were from Greiner (Stonehouse, UK). All reagents were from Sigma (Poole, UK) unless otherwise stated.

Cell lines Several conditionally immortalised human cell lines were used. These were derived by incorporating a temperaturesensitive SV40 gene, which enables cells to proliferate at the permissive temperature $\left(33^{\circ} \mathrm{C}\right)$ and to differentiate at the nonpermissive temperature $\left(37^{\circ} \mathrm{C}\right)$. The characteristics of the wildtype human podocyte cell line have been reported [19]. The cells have additionally been transfected with a telomerase construct [20]. A podocyte cell line derived from a patient with Fin Major nephrin mutation was used and the characteristics of these podocytes have also been reported previously $[17,19,21]$. Cells were cultured for 14 days at the nonpermissive temperature $\left(37^{\circ} \mathrm{C}\right)$ in RPMI 1640 medium with glutamine (R-8758; Sigma, St Louis, MO, USA) supplemented with $10 \%$ (vol./vol.) FCS (Life Technologies, Grand Island, NY, USA) and insulin transferrin sodium selenite (Sigma I-1184; 1\% [vol./vol.]). Conditionally immortalised human glomerular endothelial cells were also used. The characteristics of these cells have been reported elsewhere [22]. In addition, murine 3T3-L1 fibroblast clones were obtained from LGC Promochem (Teddington, UK). They were differentiated to adipocytes as previously described [23]. Finally, an immortalised human proximal tubular epithelial cell line (HK2) was used [24].

Stimulation of cells Cells were stimulated for $24-48 \mathrm{~h}$ with rosiglitazone (donated by GlaxoSmithKline). Rosiglitazone was dissolved in DMSO, $5 \mathrm{mg}$ in $1 \mathrm{ml}$, and added to RPMI to give a final concentration of $10 \mu \mathrm{mol} / 1$ and $0.01 \%$ (vol./ vol.) DMSO. The vehicle control for rosiglitazone was RPMI with $0.01 \%$ (vol./vol.) DMSO (termed vehicle). The dose of rosiglitazone was taken from previously published studies of cells in vitro $[25,26]$. For palmitate treatment, podocytes were stimulated for $24 \mathrm{~h}$ with $750 \mu \mathrm{mol} / \mathrm{l}$ palmitate. The preparation of media containing palmitate was based on a previously published method [27]. Briefly, $20 \%$ fatty-acid-free $\mathrm{BSA}$ was heated to $37^{\circ} \mathrm{C}$ before the addition of palmitate dissolved in ethanol. The solution was heated to $37^{\circ} \mathrm{C}$ until clear and diluted with RPMI to give a final concentration of $5 \%$ (wt/vol.) BSA, $750 \mu \mathrm{mol} / 1$ palmitate and $1 \%$ (vol./vol.) ethanol. Solutions were filter sterilised and, if required, rosiglitazone was added before cells were incubated in appropriate solutions. The control for palmitate was $5 \%$ (wt/vol.) BSA and $1 \%$ (vol./vol.) ethanol 
(termed BSA). The dose of palmitate was based on previously published studies [27, 28]

2-Deoxyglucose uptake assay The methods used were as previously reported [17]. Briefly, cells in six-well plates were pre-incubated accordingly before being serum starved in medium containing the required treatment for $2 \mathrm{~h}$. For the final $15 \mathrm{~min}$, cells were incubated in pre-warmed KrebsRinger phosphate buffer after washing three times in PBS. Where appropriate, cells were then treated with insulin $(100 \mathrm{nmol} / \mathrm{l})$ for $15 \mathrm{~min}$ at $37^{\circ} \mathrm{C}$. Glucose uptake was quantified by exposing the cells to 2-deoxy-D- $\left[{ }^{3} \mathrm{H}\right]$ glucose at a concentration of $50 \mu \mathrm{mol} / \mathrm{l}(37 \mathrm{kBq} / \mathrm{ml})$ for $5 \mathrm{~min}$. At the end of this incubation, the cells were washed rapidly three times in ice-cold PBS and lysed in $0.5 \mathrm{ml}$ of PBS containing $1 \%$ (vol./vol.) Triton X-100. The cell-associated $\beta$ emissions were then determined by liquid-scintillation counting. All experiments were carried out in triplicate.

GLUT1 small interfering RNA transfection As previously reported [17], a combination of two sequences was used with the following sense target sequences: CCAAGAGUGUG CUAAAGAAUU and CAUCGUGGCUGAACUCUUCUU. In addition, a non-specific control small interfering RNA (siRNA) sequence was used (Dharmacon, Lafayette, CO, USA). Cells were transfected using Lipofectamine 2000 (Invitrogen, Paisley, UK). Lipofectamine and siRNA were separately diluted in serum-free RPMI and the two solutions were combined and allowed to stand for $30 \mathrm{~min}$ before being added to cells in normal culture conditions. The final concentration of siRNA was $60 \mathrm{nmol} / \mathrm{l}$. After $48 \mathrm{~h}$, cells were serum starved for $2 \mathrm{~h}$ for 2-deoxyglucose-uptake experiments.

Bis-mannose photolabelling Bis-mannose labelling is a method whereby bis-labelled mannose is covalently linked to cell-surface glucose transporters which can then be detected and quantified by western blotting. This method was performed as previously described [17, 29], but with some modifications. Briefly, stimulated podocytes were incubated with and without $100 \mathrm{nmol} / 1$ insulin for $12 \mathrm{~min}$ and then incubated for a further $3 \mathrm{~min}$ in the presence of $200 \mu \mathrm{mol} / \mathrm{l}$ PEG-biotincap-ATB-BMPA (Toronto Research Chemical, Toronto, ON, Canada). Cells were then irradiated for $1 \mathrm{~min}$ in a CL-1000M crosslinker (UVP, Cambridge, UK) using $302 \mathrm{~nm}$ wavelength lamps. Cell protein concentration was quantified using an assay based on the bicinchoninic acid assay (Pierce, Rockford, IL, USA) and protein was extracted for western blotting.

Western blotting For whole-cell lysates, cells were washed twice with ice-cold PBS and then scraped into ice-cold NP40 extraction buffer $(50 \mathrm{mmol} / \mathrm{l}$ Tris $/ \mathrm{HCl}, \mathrm{pH} 7.5$, containing $1 \mathrm{mmol} / \mathrm{l}$ EDTA, $120 \mathrm{mmol} / \mathrm{l} \mathrm{NaCl}, 50 \mathrm{mmol} / \mathrm{l} \mathrm{NaF}$,
$1 \mathrm{mmol} / \mathrm{l}$ benzamidine, $1 \%$ (vol./vol.) NP40, $1 \mu \mathrm{mol} / 1$ microcystin, $7.2 \mathrm{mmol} / 12$-mercaptoethanol, $5 \mathrm{mmol} / \mathrm{l}$ orthovanadate and $1 \mu \mathrm{l} / \mathrm{ml}$ protease inhibitor cocktail (Sigma)). Cell extracts were centrifuged at $10000 \mathrm{~g}$ for $10 \mathrm{~min}$ at $4^{\circ} \mathrm{C}$ and the supernatant fractions were saved. Whole-cell membrane fractions were prepared as previously described [30] with the final membrane pellet being resuspended in NP40 extraction buffer. SDS PAGE using 7.5\% (wt/vol.) acrylamide gels was performed and protein was transferred onto polyvinylidene fluoride membranes (Millipore, Billerica, MA, USA). Membranes were blocked with $5 \%$ (wt/vol.) BSA and then incubated with the following primary antibodies: rabbit anti-PPAR $\gamma$ (Santa Cruz SC-7196; Santa Cruz Biotechnology, Santa Cruz, CA, USA), rabbit polyclonal anti-GLUT1 (a kind gift from G. Holman, Bath University, Bath, UK) and anti-nephrin antibody 50A9 (a kind gift from K. Tryggvason, Karolinska Institute, Stockholm, Sweden). Secondary horseradish-peroxidase-conjugated antibodies (Amersham Biosciences, Little Chalfont, UK) were used and luminescence was created with Femto Supersignal luminal (Pierce) before imaging in a ChemiDoc-it imaging system (UV Products, Cambridge, UK).

Immunofluorescence Immunofluorescence of cells was performed using a modified protocol as previously described [19]. Podocytes on cover slips were stimulated accordingly, washed with ice-cold PBS and then fixed with $2 \%$ (vol./vol.) paraformaldehyde. Cells were permeabilised and blocked with $0.1 \%$ (vol./vol.) saponin and 3\% (wt/vol.) BSA in PBS. They were incubated with anti-GLUT1 (a kind gift from G. Holman, Bath University, UK) and FITC secondary antibody in the blocking solution. Cells were imaged using an inverted fluorescence microscope (AF6000LX; Leica Microsystems, Mannheim, Germany). GLUT1 images of wild-type podocytes were scored by two independent blinded observers who scored at least 100 cells per condition for cytoplasmic or peripheral localisation.

Statistical analysis ANOVA was performed for glucose-uptake assays and densitometry and the groups were compared with a post hoc Bonferroni multiple comparison test. For the GLUT1 scoring an unpaired $t$ test was used. GraphPad Prism, Version 4.00, was used for analysis (GraphPad Software, San Diego, CA, USA). Significance was deemed to occur at $p<0.05$. The standard error of the mean is shown for all experiments.

\section{Results}

Production of PPAR $\gamma$ in cultured podocytes Production of PPAR $\gamma$ was confirmed by western blotting in proliferating and differentiated podocytes (Fig. 1). 


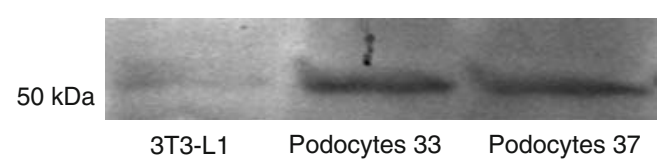

Fig. 1 Podocyte production of PPAR $\gamma$ was confirmed by western blotting. PPAR $\gamma$ was produced in undifferentiated podocytes at $33^{\circ} \mathrm{C}$ (Podocytes 33), differentiated podocytes at $37^{\circ} \mathrm{C}$ (Podocytes 37) and also in control adipocytes (3T3-L1)

Rosiglitazone increased glucose uptake in podocytes and adipocytes Rosiglitazone significantly increased basal and insulin-stimulated glucose uptake in podocytes (Fig. 2a). This effect was greatest at $48 \mathrm{~h}$ and at this time point the increase in basal glucose uptake was equivalent in magnitude to the glucose uptake seen with insulin stimulation. Basal glucose uptake in 3T3-L1 adipocytes was also significantly increased at $24 \mathrm{~h}$ following rosiglitazone treatment (Fig. 2b) though this increase was of a lesser
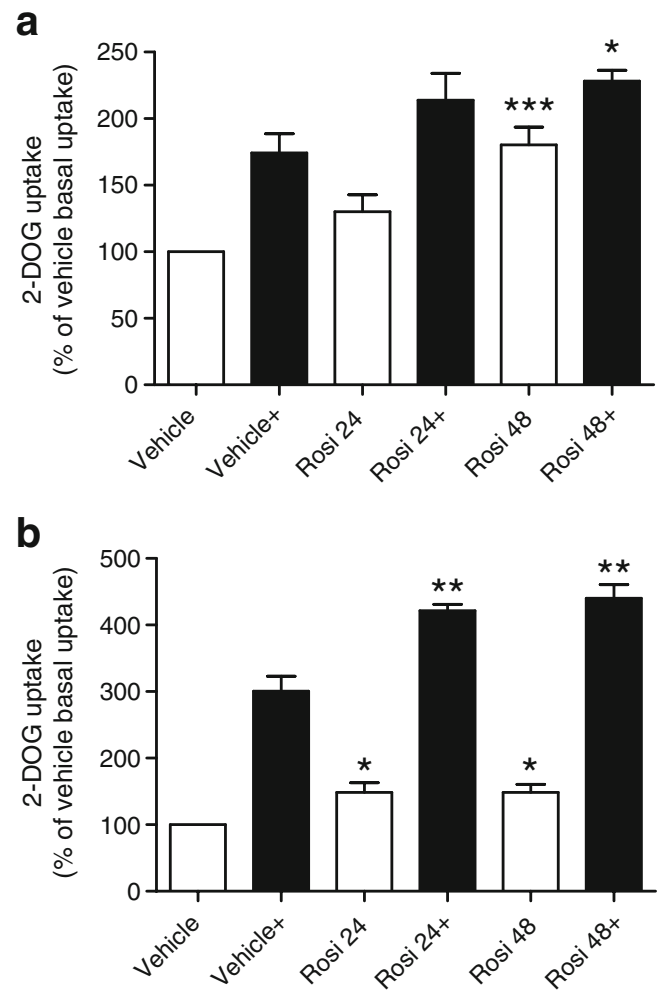

Fig. 2 a Rosiglitazone (Rosi) significantly increased basal and insulin-stimulated $(+)$ glucose uptake in podocytes at $48 \mathrm{~h}$ (one-way ANOVA $p<0.0001$ with post hoc Bonferroni ${ }^{* * *} p<0.001$ and ${ }^{*} p<$ 0.05). Data are expressed as the percentage of vehicle basal uptake and the SEM is shown of six independent experiments, each performed in triplicate. b In 3T3-L1 adipocytes, rosiglitazone significantly increased basal and insulin-stimulated $(+)$ glucose uptake at 24 and $48 \mathrm{~h}$ (one-way ANOVA comparing basal $p=0.0095$ with post hoc Bonferroni ${ }^{*} p<0.05$ and comparing insulin-stimulated $p=$ 0.0003 with post hoc Bonferroni ${ }^{* *} p<0.01$ ). Data are expressed as percentage of vehicle basal uptake and the SEM is shown of three independent experiments, each performed in triplicate. 2-DOG, 2deoxyglucose magnitude than that in podocytes. In these adipocytes, insulin-stimulated glucose uptake in adipocytes was also enhanced by rosiglitazone, as previously reported [31]. Rosiglitazone did not increase basal glucose uptake in either human glomerular endothelial cells (Fig. 3a) or human proximal tubular (HK2) cells (Fig. 3b) after $24 \mathrm{~h}$.

The increased glucose uptake in podocytes treated with rosiglitazone was abolished with GLUT1 siRNA GLUT1 is the predominant glucose transporter responsible for glucose uptake under basal conditions in podocytes. We previously used GLUT1-specific siRNA and demonstrated GLUT1 knockdown in differentiated podocytes by western blotting at $48 \mathrm{~h}$ with no change in GLUT4 production [17]. In this study the increased basal glucose uptake that occurred in podocytes with rosiglitazone was significantly reduced with GLUT1 siRNA (Fig. 4). Vehicle-treated cells also demonstrated reduced basal glucose uptake following GLUT1 siRNA, consistent with GLUT 1 being the principal transporter for basal uptake. GLUT1 siRNA altered insulin-stimulated glucose uptake in both vehicle- and rosiglitazone-treated cells compared with controls. In the same experiment, rosiglitazone significantly increased basal glucose uptake in podocytes treated with and without control GLUT1 siRNA. These findings support a GLUT1dependent mechanism for the increased glucose uptake seen in podocytes treated with rosiglitazone.
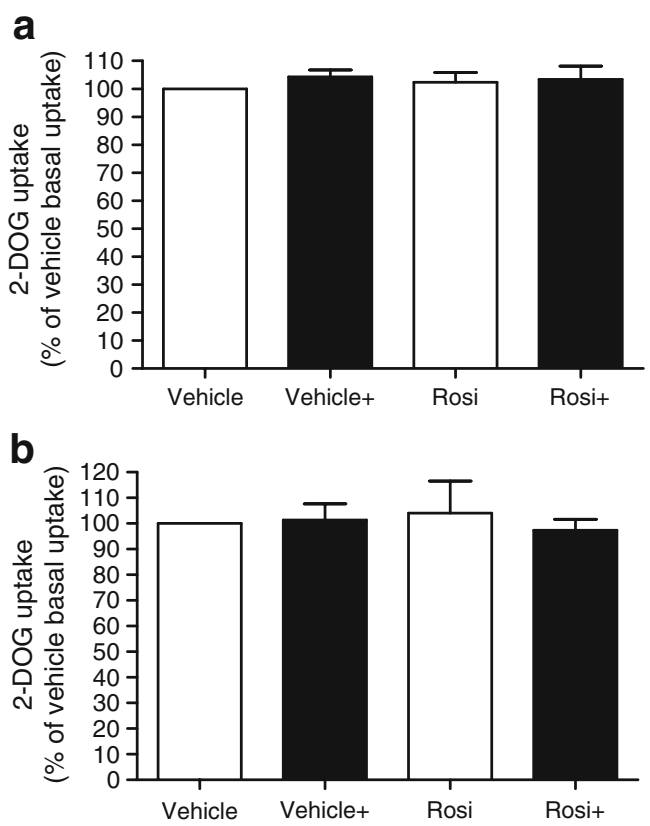

Fig. 3 There was no increase in basal or insulin-stimulated $(+)$ glucose uptake in either human glomerular endothelial cells (a) or proximal tubular (HK2) cells (b) treated with rosiglitazone (Rosi) for $24 \mathrm{~h}$. Data are expressed as percentage of vehicle basal uptake and the SEM is shown of three to eight independent experiments, each performed in triplicate. 2-DOG, 2-deoxyglucose 


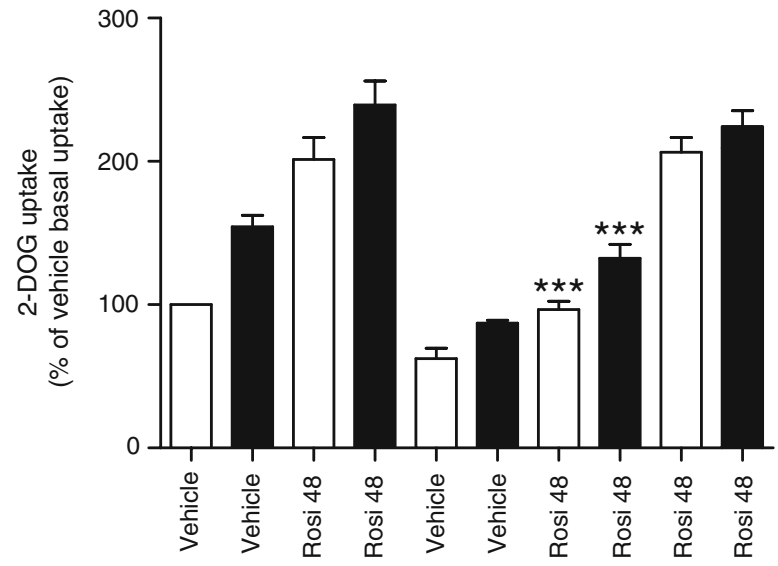

GLUT1 SiRNA $\quad-\quad-\quad-\quad-\quad+\quad+\quad+\quad+-$

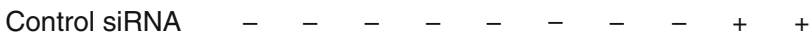
Insulin

Fig. 4 Podocytes treated with rosiglitazone or rosiglitazone with control siRNA demonstrated a significant increase in basal and insulin-stimulated glucose uptake. This effect was significantly blocked by GLUT1 siRNA (one-way ANOVA with post hoc Bonferroni $\left.{ }^{* * *} p<0.001\right)$. Data are expressed as percentage of vehicle basal uptake and the SEM is shown of three experiments. 2-DOG, 2deoxyglucose

Nephrin-deficient podocytes did not demonstrate increased glucose uptake with rosiglitazone We proceeded to investigate the functional role of nephrin. Nephrin is a key component of the podocyte slit diaphragm protein complex. In keeping with our previous findings [18], we found in this study that nephrin-deficient podocytes derived from a patient with a Fin Major nephrin mutation did not increase glucose uptake in response to insulin (Fig. 5a). Furthermore, we found that rosiglitazone did not increase glucose uptake in nephrin-deficient podocytes.

Nephrin production was unchanged in wild-type podocytes treated with rosiglitazone The production of nephrin therefore appears critical for the increased glucose uptake observed in podocytes treated with rosiglitazone. However, we found there was no difference in nephrin production in wild-type podocytes treated with rosiglitazone for $24-48 \mathrm{~h}$ (Fig. 5b) and this is in keeping with results from a recent in vivo study [16].

Rosiglitazone increased the translocation of GLUT1 to the plasma membrane in wild-type podocytes We further examined the role of GLUT1. Podocytes treated with rosiglitazone for $48 \mathrm{~h}$ had increased peripheral localisation of GLUT1 on immunostaining compared with vehicletreated cells (Fig. 6). By comparison, GLUT1 translocation to the plasma membrane was reduced in nephrin-deficient podocytes. This finding is consistent with our previously published data demonstrating that nephrin-deficient podocytes were unable to translocate GLUT1 to the plasma membrane following insulin stimulation [18].

In addition, we found that membrane fractions from wild-type podocytes treated with rosiglitazone demonstrated increased GLUT1 compared with cytoplasmic fractions and vehicle-treated controls (Fig. 7a). To further validate these findings an assay of functional glucose transporters was used. Bis-mannose photolabelling of cell surface glucose transporters demonstrated increased GLUT1 at the plasma membrane in podocytes treated with rosiglitazone (Fig. 7b). There was no difference in the total cellular GLUT1 production between vehicle- and rosiglitazonetreated podocytes (Fig. 7C) and this is in keeping with findings from other studies [16, 32].

Rosiglitazone increased glucose uptake in podocytes treated with palmitate Palmitate is the predominant circulating saturated fatty acid and it is increased in insulinresistant states such as type 2 diabetes [33]. We used palmitate to induce insulin resistance in podocytes. Treatment of podocytes with palmitate for $24 \mathrm{~h}$ resulted in

a

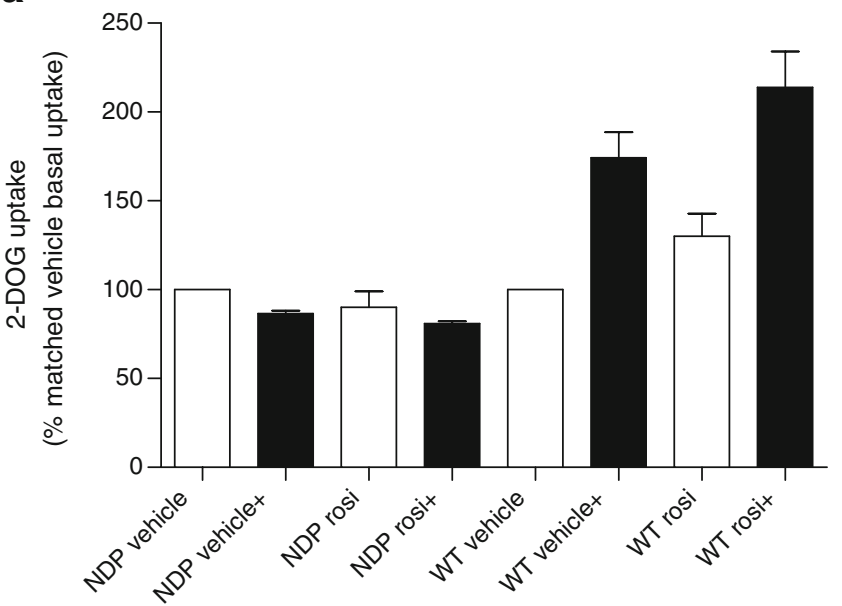

b

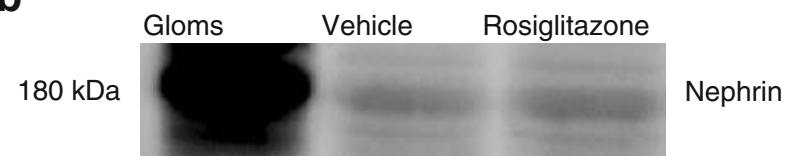

$37 \mathrm{kDa}$

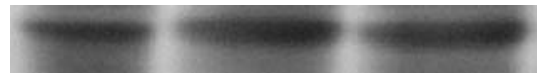

$\beta$-Actin

Fig. 5 a Unlike wild-type (WT) podocytes, there were no differences in glucose uptake in nephrin-deficient podocytes (NDP) treated with insulin $(+)$ or rosiglitazone (rosi). Data are expressed as percentage of vehicle basal uptake and the SEM is shown of three experiments. b There was no change in nephrin expression in wild-type podocytes treated with rosiglitazone. This blot is representative of three independent experiments. 2-DOG, 2-deoxyglucose; Gloms, glomeruli 

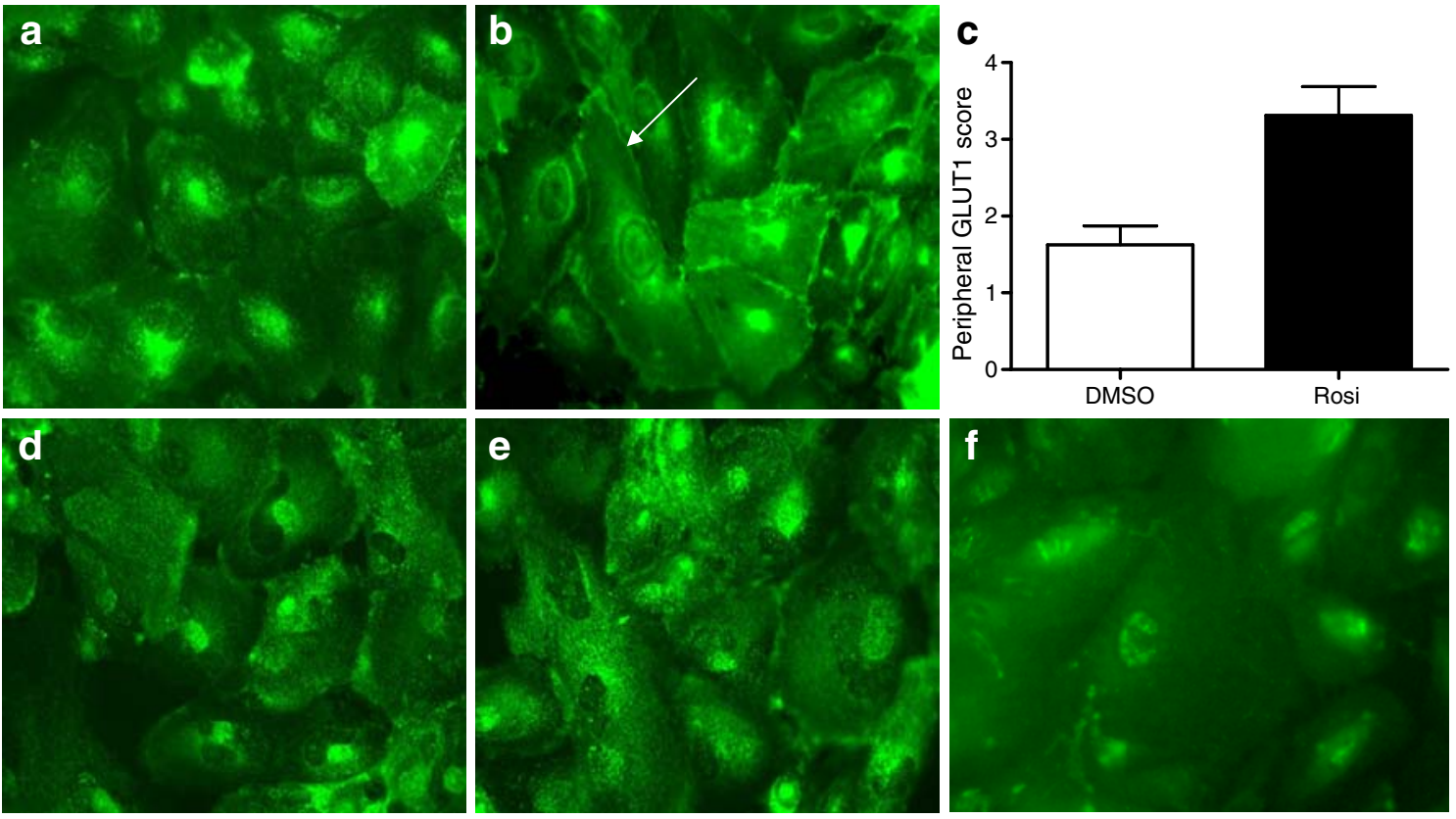

Fig. 6 Differentiated wild-type podocytes were treated with a $0.01 \%$ (vol./vol.) DMSO (vehicle) or b $10 \mu \mathrm{mol} / 1$ rosiglitazone for $48 \mathrm{~h}$. There was a consistent increase in peripheral GLUT1 in rosiglitazonetreated cells (arrow) compared with vehicle-treated cells. This was scored by two blinded independent observers (c) who found a significant increase $(p=0.0022)$ in peripheral GLUT1 localisation in wild-type cells treated with rosiglitazone (Rosi). By contrast, compared with those treated with vehicle (d), there was no change in the localisation of GLUT1 in nephrin-deficient podocytes treated with rosiglitazone (e). The diffuse cytoplasmic staining seen in vehicle-treated cells was not seen with rabbit $\operatorname{IgG}$ staining (f)

effect of rosiglitazone on increasing basal glucose uptake was, therefore, preserved in podocytes treated with palmitate and this led to a corresponding increase in insulinstimulated glucose uptake. This may represent a mechanism by which rosiglitazone preserves podocyte function in insulin-resistant states.

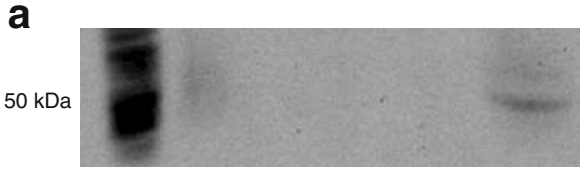

3T3-L1 Vehicle Vehicle (M) Rosi Rosi (M)
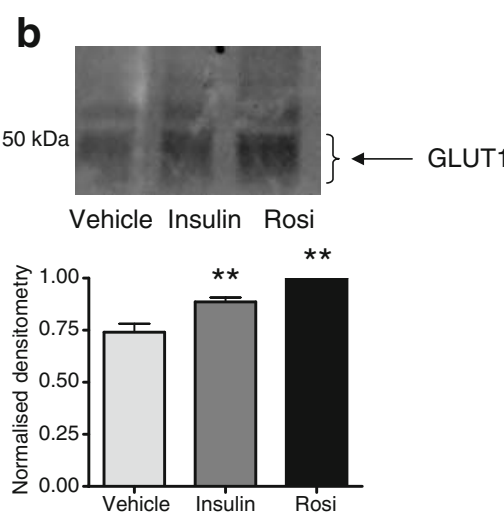

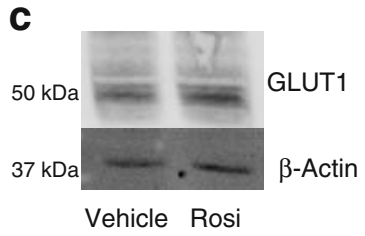

labelling. Podocytes treated with rosiglitazone $(10 \mu \mathrm{mol} / \mathrm{l})$ or insulin (100 nmol/1 for $15 \mathrm{~min})$ had significantly increased GLUT1 production compared with vehicle-treated cells $\left({ }^{* *} p<0.01\right)$. c There was no change in total cellular GLUT1 in podocytes treated with rosiglitazone. All blots shown are representative of three to five independent experiments
Fig. 7 Differentiated wild-type podocytes were treated with $0.01 \%$ (vol./vol.) DMSO (vehicle) or $10 \mu \mathrm{mol} / 1$ rosiglitazone (Rosi) for $48 \mathrm{~h}$. a In membrane preparations (M) of these cells there was increased expression of GLUT1 with rosiglitazone treatment compared with vehicle or cytoplasmic fractions. Whole-cell insulin-stimulated adipocytes (3T3-L1) were used as a positive control. b The cell-surface expression of GLUT1 was determined using bis-mannose photo- 


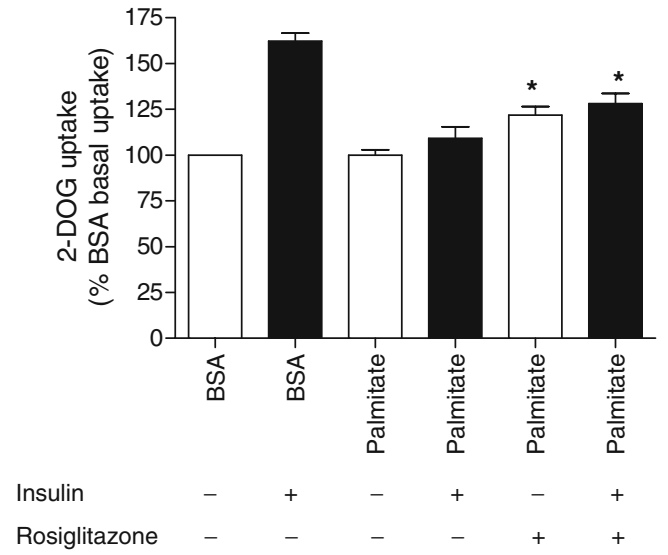

Fig. 8 Cells were cultured in six-well plates supplemented with 5\% (wt $/ \mathrm{vol}$.) BSA or $750 \mu \mathrm{mol} / 1$ palmitate with or without rosiglitazone $(10 \mu \mathrm{mol} / \mathrm{l})$ for $24 \mathrm{~h}$ followed by $2 \mathrm{~h}$ in the corresponding serum-free medium. 2-Deoxyglucose (2-DOG) uptake over $5 \mathrm{~min}$ was measured following stimulation with or without $100 \mathrm{nmol} / 1$ insulin for $15 \mathrm{~min}$. Podocytes treated with palmitate had significantly reduced insulinstimulated glucose uptake. Co-treatment with rosiglitazone and palmitate significantly increased both basal and insulin-stimulated glucose uptake by comparison with palmitate alone $\left({ }^{*} p<0.05\right)$, but uptake did not fully recover to the insulin-stimulated uptake at $24 \mathrm{~h}$. Data are expressed as percentage of vehicle basal uptake and the SEM is shown of five independent experiments, each performed in triplicate

\section{Discussion}

The role PPAR $\gamma$ agonists may play in the prevention and treatment of renal disease is emerging. These agents have recognised insulin-sensitising properties and therefore have an important role in the treatment of type 2 diabetes and insulin resistance. The reduction in microalbuminuria observed in patients with type 2 diabetes treated with PPAR $\gamma$ agonists raises important questions about the potential mechanisms by which these agents work.

The importance of podocytes in maintaining the integrity of the glomerular filtration barrier was encapsulated by the discovery of the key podocyte protein, nephrin, in 1998. Mutations in NPHS1, which encodes nephrin, result in congenital nephrotic syndrome of the Finnish type [34]. Since the discovery of nephrin, a number of genes have been found to be mutated in human nephrotic syndromes or animal models and these discoveries have provided significant insights into disease mechanisms [35].

This study reports a direct functional effect of rosiglitazone on podocytes. After treatment for $48 \mathrm{~h}$, rosiglitazone significantly increased basal glucose uptake and this effect has been observed in other insulin-sensitive cells [31]. This effect was not seen in glomerular endothelial cells suggesting that, at this functional level, rosiglitazone is having an effect on one component of the glomerular filtration barrier. As rosiglitazone had an insulin-sensitising effect in podo- cytes and adipocytes, the role of the glucose transporter GLUT1 was investigated as a potential mechanism for the observed increase in both basal and insulin-stimulated glucose uptake.

GLUT1 is the predominant basal glucose transporter and we have previously reported GLUT1 production in podocytes, as well as a role for GLUT1 in insulin-mediated glucose transport [17]. Using GLUT1 siRNA it was possible to block the increase in glucose uptake in podocytes treated with rosiglitazone. The increase in glucose uptake was not abolished completely, which may be explained by incomplete knockdown or by the contribution of other glucose transporters. We have demonstrated the presence of GLUT2 in podocytes (M. A. Saleem, unpublished data) and GLUT8 has also been reported to have high levels of production in the kidney [36].

In support of these functional findings, we found increased peripherally localised and membrane-associated GLUT1. Furthermore, there was increased cell-surface expression of GLUT1 associated with rosiglitazone treatment whereas total cellular GLUT1 was unchanged. Increased production of GLUT1 has been associated with diabetic nephropathy, although this may not be a feature of early disease or animal models [16]. In line with our findings, a study reported increased membrane production of GLUT1 in adipocytes following incubation with rosiglitazone without a change in total GLUT1 [32].

In our study, the role of nephrin was investigated with nephrin-deficient podocytes derived from a patient with a Fin Major nephrin mutation. The increased basal glucose uptake seen with wild-type podocytes treated with rosiglitazone was absent in nephrin-deficient cells and in addition these cells did not respond to insulin as previously reported [18]. Furthermore, there was limited peripheral localisation of GLUT1 in nephrin-deficient podocytes following treatment with rosiglitazone.

These findings indicate a critical role for nephrin in mediating the increased glucose uptake in podocytes treated with rosiglitazone. Nephrin has also been associated with diabetic nephropathy, with reduced nephrin levels in human biopsies from patients with diabetes and proteinuria [12]. It has been proposed that this contributes to the development of nephropathy. Therefore, restoration of nephrin production could be a mechanism to reduce glomerular filtration barrier dysfunction in proteinuric states, such as diabetic nephropathy. Indeed pioglitazone, which has been shown to reduce microalbuminuria in diabetic nephropathy [7], has also been shown to restore nephrin mRNA and protein levels in a Heymann nephritis model [13]. This study also demonstrated that pioglitazone regulates the expression of the gene encoding nephrin.

In our study, $48 \mathrm{~h}$ incubation with rosiglitazone did not increase the production of nephrin in wild-type podocytes. 
This may have been due to the duration of rosiglitazone treatment or to normal regulation of nephrin production. It is plausible that nephrin production is maintained within limits for sufficiency. Our finding is also in keeping with a recent study that did not demonstrate changes in glomerular nephrin, GLUT1 and Neph1 (also known as Kirrel) expression in diabetic mice following either 10 or 20 weeks of rosiglitazone treatment [16].

Finally, to draw analogy to a disease state, we used the saturated NEFA palmitate. This blocked insulin-stimulated glucose uptake in wild-type podocytes but did not block the effect of rosiglitazone on podocyte glucose uptake. There was a significant increase in basal glucose uptake in cells co-treated with palmitate and rosiglitazone compared with palmitate alone and this was associated with a corresponding increase in insulin-stimulated glucose uptake.

We have also demonstrated that palmitate interrupts the insulin signalling pathway leading to GLUT4 translocation [37] and we propose that this mechanism is involved in the development of nephropathy in insulin-resistant states. Since the effect of rosiglitazone was preserved in cells treated with palmitate, we suggest that is a means by which $\operatorname{PPAR} \gamma$ agonists are protective in nephropathy associated with insulin resistance.

In addition to our study, other studies have reported direct effects of PPAR $\gamma$ agonists on podocytes. Pioglitazone was shown to be protective in a puromycin aminonucleoside (PAN) model of podocyte injury by reducing podocyte apoptosis [14]. Furthermore, the related in vivo study demonstrated a reduction in glomerulosclerosis in PAN-injured rats following treatment with pioglitazone [15]. PPAR $\gamma$ agonists, therefore, have diverse effects on podocyte function and it will be important to further define the pathways involved.

In conclusion, we have shown a direct functional effect of rosiglitazone on podocytes and we have demonstrated that the mechanism occurs via an effect on GLUT1 trafficking. Although we have not demonstrated the mechanism of GLUT1 translocation in this study, we have previously shown that it is dependent upon nephrin and actin in podocytes [18]. We propose that podocytes have a high metabolic demand and as such they require a ready supply of energy substrates. Insulin-resistant states such as type 2 diabetes may adversely affect this substrate provision. Clinically, PPAR $\gamma$ agonists confer renal protection and the functional effect that we have observed may be involved in the mechanism of protection. If PPAR $\gamma$ agonists are protective against progression of renal disease across the spectrum of insulin resistance [38, 39], there are potentially more applications for this group of drugs. The effect of these drugs in reducing the progression of renal disease requires further investigation.
Acknowledgements R. Lennon was funded by the Wellcome Trust for this work. A. Singh was funded by Kidney Research UK. R. J. Coward is funded by the Medical Research Council. Additional funding was received from the Nephrotic Syndrome Trust and we thank North Bristol NHS Trust for funding which also supported this work. We also thank M. A. Sabin and J. P. Shield for their advice regarding palmitate stimulation.

Duality of interest The authors declare that there is no duality of interest associated with this manuscript.

\section{References}

1. Issemann I, Green S (1990) Activation of a member of the steroid hormone receptor superfamily by peroxisome proliferators. Nature 347:645-650

2. Desvergne B, Wahli W (1999) Peroxisome proliferator-activated receptors: nuclear control of metabolism. Endocr Rev 20:649-688

3. Kersten S, Desvergne B, Wahli W (2000) Roles of PPARs in health and disease. Nature 405:421-424

4. Campbell IW (2005) The clinical significance of PPAR gamma agonism. Curr Mol Med 5:349-363

5. Bhatia V, Viswanathan P (2006) Insulin resistance and PPAR insulin sensitizers. Curr Opin Investig Drugs 7:891-897

6. Gerstein HC, Yusuf S, Bosch J et al (2006) Effect of rosiglitazone on the frequency of diabetes in patients with impaired glucose tolerance or impaired fasting glucose: a randomised controlled trial. Lancet 368:1096-1105

7. Nakamura T, Ushiyama C, Osada S, Hara M, Shimada N, Koide H (2001) Pioglitazone reduces urinary podocyte excretion in type 2 diabetes patients with microalbuminuria. Metabolism 50:1193-1196

8. Imano E, Kanda T, Nakatani Y et al (1998) Effect of troglitazone on microalbuminuria in patients with incipient diabetic nephropathy. Diabetes Care 21:2135-2139

9. Panchapakesan U, Chen XM, Pollock CA (2005) Drug insight: thiazolidinediones and diabetic nephropathy-relevance to renoprotection. Nat Clin Pract Nephrol 1:33-43

10. Zhang Y, Guan Y (2005) PPAR-gamma agonists and diabetic nephropathy. Curr Diab Rep 5:470-475

11. Nakamura T, Ushiyama C, Suzuki S et al (2000) Urinary excretion of podocytes in patients with diabetic nephropathy. Nephrol Dial Transplant 15:1379-1383

12. Doublier S, Salvidio G, Lupia E et al (2003) Nephrin expression is reduced in human diabetic nephropathy: evidence for a distinct role for glycated albumin and angiotensin II. Diabetes 52:1023-1030

13. Benigni A, Zoja C, Tomasoni S et al (2006) Transcriptional regulation of nephrin gene by peroxisome proliferator-activated receptor- $\gamma$ agonist: molecular mechanism of the antiproteinuric effect of pioglitazone. J Am Soc Nephrol 17:1624-1632

14. Kanjanabuch T, Ma LJ, Chen J et al (2007) PPAR-gamma agonist protects podocytes from injury. Kidney Int 71:1232-1239

15. Yang HC, Ma LJ, Ma J, Fogo AB (2006) Peroxisome proliferatoractivated receptor-gamma agonist is protective in podocyte injuryassociated sclerosis. Kidney Int 69:1756-1764

16. Zhang H, Saha J, Byun J et al (2008) Rosiglitazone reduces renal and plasma markers of oxidative injury and reverses urinary metabolite abnormalities in the amelioration of diabetic nephropathy. Am J Physiol Renal Physiol 295:F1071-F1081

17. Coward RJ, Welsh GI, Yang J et al (2005) The human glomerular podocyte is a novel target for insulin action. Diabetes 54:3095-3102

18. Coward RJ, Welsh GI, Koziell A et al (2007) Nephrin is critical for the action of insulin on human glomerular podocytes. Diabetes $56: 1127-1135$ 
19. Saleem MA, O'Hare MJ, Reiser J et al (2002) A conditionally immortalized human podocyte cell line demonstrating nephrin and podocin expression. J Am Soc Nephrol 13:630-638

20. O'Hare MJ, Bond J, Clarke C et al (2001) Conditional immortalization of freshly isolated human mammary fibroblasts and endothelial cells. Proc Natl Acad Sci U S A 98:646-651

21. Coward RJ, Foster RR, Patton D et al (2005) Nephrotic plasma alters slit diaphragm-dependent signaling and translocates nephrin, Podocin, and CD2 associated protein in cultured human podocytes. J Am Soc Nephrol 16:629-637

22. Satchell SC, Tasman CH, Singh A et al (2006) Conditionally immortalized human glomerular endothelial cells expressing fenestrations in response to VEGF. Kidney Int 69:1633-1640

23. Oatey PB, Van Weering DH, Dobson SP, Gould GW, Tavare JM (1997) GLUT4 vesicle dynamics in living 3T3 L1 adipocytes visualized with green-fluorescent protein. Biochem J 327:637-642

24. Ryan MJ, Johnson G, Kirk J, Fuerstenberg SM, Zager RA, TorokStorb B (1994) HK-2: an immortalized proximal tubule epithelial cell line from normal adult human kidney. Kidney Int 45:48-57

25. Kumar N, Dey CS (2003) Development of insulin resistance and reversal by thiazolidinediones in $\mathrm{C} 2 \mathrm{C} 12$ skeletal muscle cells. Biochem Pharmacol 65:249-257

26. Sander TL, Noll L, Klinkner DB et al (2006) Rosiglitazone antagonizes vascular endothelial growth factor signaling and nuclear factor of activated $\mathrm{T}$ cells activation in cardiac valve endothelium. Endothelium 13:181-190

27. Schmitz-Peiffer C, Craig DL, Biden TJ (1999) Ceramide generation is sufficient to account for the inhibition of the insulin-stimulated $\mathrm{PKB}$ pathway in $\mathrm{C} 2 \mathrm{C} 12$ skeletal muscle cells pretreated with palmitate. J Biol Chem 274:24202-24210

28. Sabin MA, Stewart CE, Crowne EC et al (2007) Fatty acid-induced defects in insulin signalling, in myotubes derived from children, are related to ceramide production from palmitate rather than the accumulation of intramyocellular lipid. J Cell Physiol 211:244-252

29. Ryder JW, Yang J, Galuska D et al (2000) Use of a novel impermeable biotinylated photolabeling reagent to assess insulinand hypoxia-stimulated cell surface GLUT4 content in skeletal muscle from type 2 diabetic patients. Diabetes 49:647-654
30. Ladomery M, Sommerville J, Woolner S, Slight J, Hastie N (2003) Expression in Xenopus oocytes shows that WT1 binds transcripts in vivo, with a central role for zinc finger one. J Cell Sci 116:1539-1549

31. Nugent C, Prins JB, Whitehead JP et al (2001) Potentiation of glucose uptake in 3T3-L1 adipocytes by PPAR gamma agonists is maintained in cells expressing a PPAR gamma dominant-negative mutant: evidence for selectivity in the downstream responses to PPAR gamma activation. Mol Endocrinol 15:1729-1738

32. Standaert ML, Kanoh Y, Sajan MP, Bandyopadhyay G, Farese RV (2002) Cbl, IRS-1, and IRS-2 mediate effects of rosiglitazone on PI3K, PKC-lambda, and glucose transport in 3T3/L1 adipocytes. Endocrinology 143:1705-1716

33. Groop L, Ekstrand A, Forsblom C et al (1993) Insulin resistance, hypertension and microalbuminuria in patients with type 2 (noninsulin-dependent) diabetes mellitus. Diabetologia 36:642-647

34. Kestila M, Lenkkeri U, Mannikko M et al (1998) Positionally cloned gene for a novel glomerular protein - nephrin - is mutated in congenital nephrotic syndrome. Mol Cell 1:575-582

35. Patari-Sampo A, Ihalmo P, Holthofer H (2006) Molecular basis of the glomerular filtration: nephrin and the emerging protein complex at the podocyte slit diaphragm. Ann Med 38:483-492

36. Schiffer M, Susztak K, Ranalletta M, Raff AC, Bottinger EP, Charron MJ (2005) Localization of the GLUT8 glucose transporter in murine kidney and regulation in vivo in nondiabetic and diabetic conditions. Am J Physiol Renal Physiol 289:F186F193

37. Lennon R, Pons D, Sabin MA et al (2009) Saturated fatty acids induce insulin resistance in human podocytes. Nephrol Dial Transplant (in press)

38. Blackwell VC, Salis P, Groves RW, Baldeweg SE, Conway GS, Unwin RJ (2001) Partial lipodystrophy, polycystic ovary syndrome and proteinuria: a common link to insulin resistance? J R Soc Med 94:238-240

39. Dogra GK, Herrmann S, Irish AB, Thomas MA, Watts GF (2002) Insulin resistance, dyslipidaemia, inflammation and endothelial function in nephrotic syndrome. Nephrol Dial Transplant $17: 2220-2225$ 